Supplement of Biogeosciences, 18, 4265-4280, 2021 https://doi.org/10.5194/bg-18-4265-2021-supplement (C) Author(s) 2021. CC BY 4.0 License.

(c) (1)

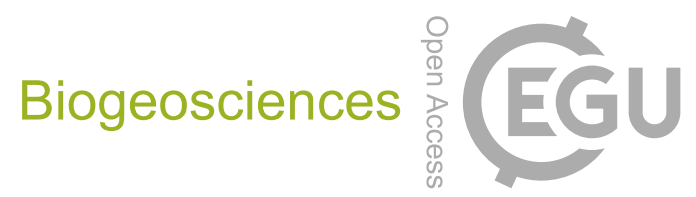

Supplement of

\title{
Seasonal cycling of zinc and cobalt in the south-eastern Atlantic along the GEOTRACES GA10 section
}

Neil J. Wyatt et al.

Correspondence to: Neil J. Wyatt (n.j.wyatt@ soton.ac.uk)

The copyright of individual parts of the supplement might differ from the article licence. 
Supplement Table S1. Regression lines as depicted in Figure 4. SAMW was identified by Si* (Sarmiento et al., 2004) with underlying AAIW located between 500 and $1750 \mathrm{~m}$. Concentrations of dissolved $\mathrm{Zn}$ and $\mathrm{Co}$ are in $\mathrm{nM}$ and $\mathrm{PO}_{4}{ }^{3-}$ in $\mu \mathrm{M}$. SAMW $=$ Sub-Antarctic Mode Water, AAIW = Antarctic Intermediate Water.

\begin{tabular}{l|l|l|c|c}
\hline Water mass & Transect & $\begin{array}{c}\text { Equation } \\
\mathrm{dZn}-\mathrm{PO}_{4}{ }^{3-} \text { relationship }\end{array}$ & $R^{2}$ & $n$ \\
\hline \multirow{2}{*}{ SAMW } & Early spring & $\mathrm{dZn}=1.4 * \mathrm{PO}_{4}{ }^{3-}-1.0$ & 0.39 & 27 \\
& Late spring & $\mathrm{dZn}=1.4 * \mathrm{PO}_{4}{ }^{3-}-0.8$ & 0.67 & 44 \\
& Summer & $\mathrm{dZn}=1.1 * \mathrm{PO}_{4}{ }^{3-}-0.7$ & 0.54 & 30 \\
AAIW & Early spring & $\mathrm{dZn}=5.8 * \mathrm{PO}_{4}{ }^{3-}-9.1$ & 0.65 & 40 \\
& Late spring & $\mathrm{dZn}=4.4 * \mathrm{PO}_{4}{ }^{3-}-6.1$ & 0.60 & 21 \\
& Summer & $\mathrm{dZn}=5.9 * \mathrm{PO}_{4}{ }^{3-}-9.4$ & 0.43 & 19 \\
\hline
\end{tabular}

\begin{tabular}{l|l|c|c|c}
\hline Water mass & Transect & $\begin{array}{c}\text { Equation } \\
\mathrm{dCo}-\mathrm{PO}_{4}{ }^{3-} \text { relationship }\end{array}$ & $R^{2}$ & $n$ \\
\hline SAMW+AAIW & Early spring & $\mathrm{dCo}=12.6 * \mathrm{PO}_{4}{ }^{3-}+28.4$ & 0.42 & 55 \\
& Late spring & $\mathrm{dCo}=15.4 * \mathrm{PO}_{4}{ }^{3-}+17.3$ & 0.67 & 56 \\
& Summer & $\mathrm{dCo}=11.4 * \mathrm{PO}_{4}{ }^{3-}+30.2$ & 0.49 & 36 \\
\hline
\end{tabular}
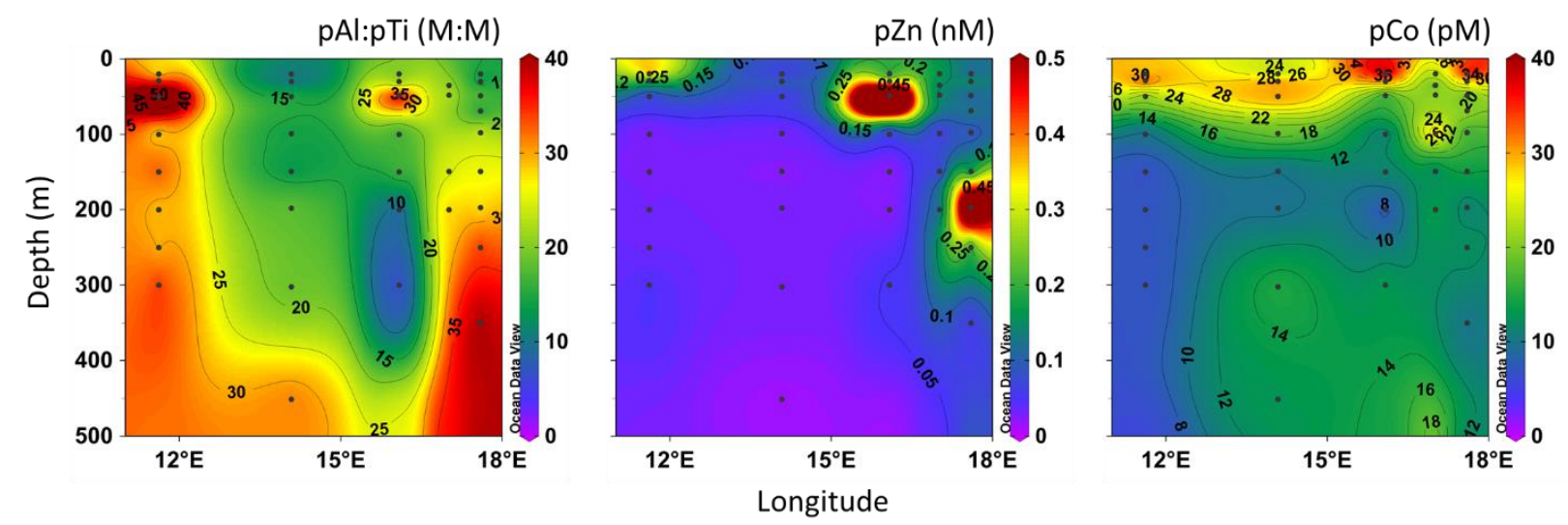

Supplement Figure S1. Upper $500 \mathrm{~m}$ distribution of particulate $\mathrm{Al}$ :Ti ratio alongside particulate $\mathrm{Zn}$ and $\mathrm{Co}$ between 11 and $18^{\circ} \mathrm{E}$ during the late spring (D357-2). 


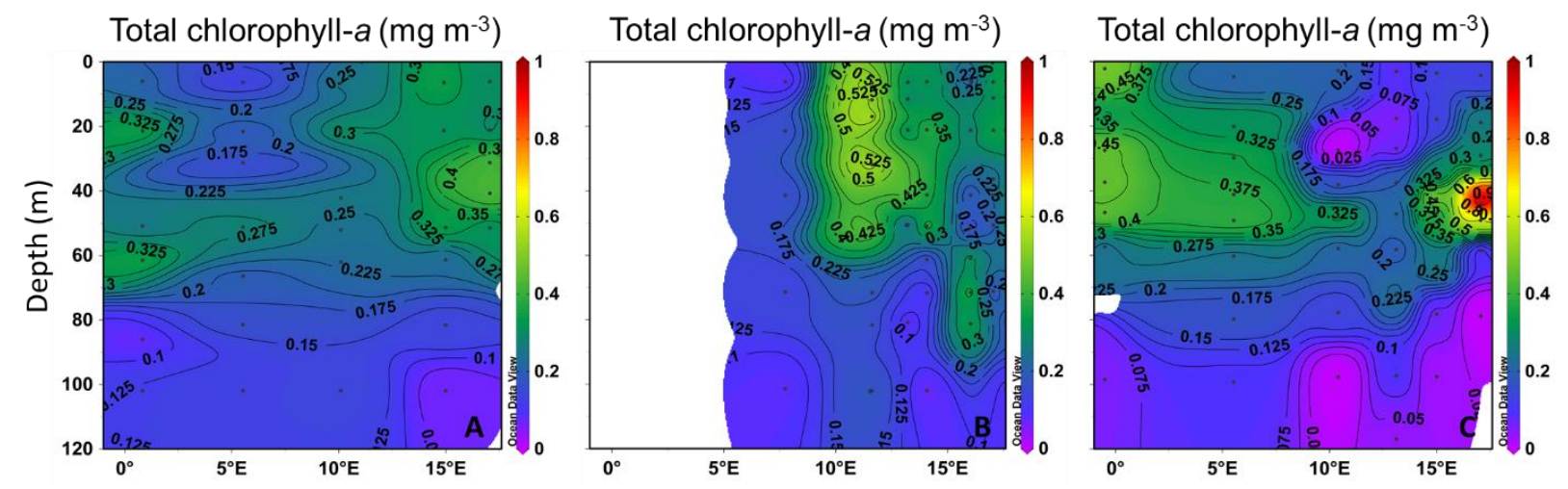

Supplement Figure S2. Upper $50 \mathrm{~m}$ distributions of total chlorophyll- $a$ during the early spring (A; D357-1), late spring (B; D357-2) and summer (C; JC068) transects. 